\title{
Manejo y tratamiento en pacientes con enfermedad por coronavirus 2019 (COVID-19) y sus implicaciones endocrinológicas
}

\section{Management and treatment in patients with coronavirus disease 2019 (COVID-19) and its endocrinological implications}

\author{
Berenice Peña-Aparicio ${ }^{1 *}$ y Javier Burgos-Cárdenas ${ }^{2}$ \\ 1Departamento de Endocrinología, Instituto Nacional de Cardiología Ignacio Chávez; ${ }^{2}$ Departamento de Anestesiología, Instituto de \\ Oftalmología, Fundación Conde de Valenciana. Ciudad de México, México
}

\section{RESUMEN}

Los casos confirmados de infección por el coronavirus 2 del síndrome respiratorio agudo grave (SARS-CoV-2) deben tratarse con condiciones efectivas de aislamiento y protección, además del control de infecciones; los casos críticos deben ingresarse a la unidad de cuidados intensivos lo antes posible. Se identifican como grupos de mayor riesgo de contagio y de COVID-19 severa, a aquellos sujetos con enfermedades crónicas, como diabetes, obesidad e hipertensión. Existe un número significativo de objetivos farmacológicos, como el ciclo vital del virus, las vías de entrada viral y la regulación inmunitaria, sin embargo, actualmente no hay evidencia procedente de ensayos clínicos aleatorizados de que cualquier terapia potencial mejore los resultados en pacientes hospitalizados con COVID-19, tampoco existen datos que respalden alguna terapia profiláctica, y mucho menos que sean inocuos. La pandemia de la enfermedad causada por el nuevo SARSCoV-2 presenta un desafío sin precedentes para el personal médico, entre ellos el endocrinólogo al enfrentarse a un nuevo reto nosológico y terapéutico.

Palabras clave: COVID-19. SARS-CoV-2. MERS. Cloroquina. Remdesivir. SDRA.

\begin{abstract}
There is currently no specific treatment for patients with coronavirus disease 2019 (COVID-19). Confirmed cases of severe acute respiratory syndrome coronavirus 2 infection (SARS-CoV-2) should be treated with effective conditions of isolation and protection, in addition to infection control; Critical cases should be admitted to the intensive care unit as soon as possible. Those subjects with chronic diseases, such as diabetes, obesity, and hypertension, are identified as groups with a higher risk of contagion and severe COVID-19. There are a significant number of pharmacological targets, such as virus life cycle, viral routes of entry, and immune regulation, however, there is currently no evidence from randomized clinical trials that any potential therapy improves outcomes in hospitalized patients with COVID. -19 , there are no data to support any prophylactic therapy, much less that they are harmless. The disease pandemic caused by the new SARSCoV-2 presents an unprecedented challenge for medical personnel, including the endocrinologist, as they face a new nosological and therapeutic challenge.
\end{abstract}

Key words: COVID-19. SARS-CoV-2. MERS. Chloroquine. Remdesivir. ARDS.
Correspondencia:

*Berenice Peña-Aparicio

E-mail: berenicepaendocrino@hotmail.com
Fecha de recepción: 06-05-2020

Fecha de aceptación: 27-05-2020

DOI: 10.24875/RME.20000034
Disponible en internet: $27-06-2020$

Rev Mex Endocrinol Metab Nutr. 2020;7:144-57

2462-4144 / @ 2020 Sociedad Mexicana de Nutricion y Endocrinologia, AC. Publicado por Permanyer. Éste es un artículo open access bajo la licencia CC BY-NC-ND (http://creativecommons.org/licenses/by-nc-nd/4.0/). 


\section{INTRODUCCIÓN}

Actualmente no hay un tratamiento específico para la enfermedad por coronavirus 2019 (COVID-19), pero dado que la mayoría de los casos son leves, solo una cantidad limitada de personas requerirá hospitalización para cuidados de apoyo. Sin embargo, en la mayoría de los países en los que se ha declarado emergencia sanitaria, particularmente en China, las regiones del norte de Italia, Irán y España, la situación ha sido muy difícil y el requisito de hospitalización ha llevado al sistema nacional de salud al límite de sus capacidades'.

El manejo más importante en los casos de pacientes afectados por la COVID-19 leve es el confinamiento en casa durante 14 días o hasta que se resuelvan los síntomas (lo que sea más largo). Individuos y familias afectadas o sospechosas de verse afectadas por la COVID-19 deben quedarse en casa y seguir correctamente las medidas para la prevención y el control de infecciones. Debe realizarse monitoreo del deterioro clínico, estar en una habitación diferente $\mathrm{o}$, si eso no es posible, mantener una distancia de al menos un metro de la persona afectada (p. ej., dormir en una cama separada) y realizar higiene de manos (lavado de manos con agua y jabón) después de cualquier tipo de contacto con la persona afectada o su entorno inmediato. Cuando se laven las manos, es preferible usar papel desechable. Si no está disponible, las toallas deben usarse y reemplazarse cuando estén mojadas. Se debe proporcionar una mascarilla médica a la persona afectada para contener las secreciones respiratorias. Las personas que no pueden tolerar una mascarilla médica deben usar higiene respiratoria rigurosa, es decir, la boca y la nariz deben cubrirse con un pañuelo desechable de papel cuando haya tos o estornudos, los cuidadores también deben usar una mascarilla médica ajustada que cubra boca y nariz cuando están en la misma habitación de la persona afectada ${ }^{1,2}$. Los casos confirmados de infección por el coronavirus 2 del síndrome respiratorio agudo grave (SARS-CoV-2) deben tratarse con condiciones efectivas de aislamiento y protección, y los casos críticos deben ingresarse en la unidad de cuidados intensivos (UCI) lo antes posible 3 .
Se identifican como grupos de mayor riesgo de contagio y de COVID-19 severa a aquellos sujetos con enfermedades crónicas, entre ellas diabetes, obesidad, enfermedades cardiovasculares e hipertensión arterial ${ }^{1}$.

\section{TRATAMIENTOS DISPONIBLES PARA LA} COVID-19

Los pasos del ciclo de vida viral proporcionan objetivos potenciales para la terapia con medicamentos. Los objetivos farmacológicos prometedores incluyen proteínas no estructurales (p. ej., proteasa tipo 3-quimotripsina, tipo papaína proteasa, ARN polimerasa dependiente de ARN), que comparten homología con otros nuevos coronavirus (CoV). Otros objetivos farmacológicos incluyen vías de entrada viral y de regulación inmunitaria. La tabla 1 resume el mecanismo de acción y los principales parámetros farmacológicos de los tratamientos seleccionados o terapias complementarias para la COVID-19. Los agentes utilizados anteriormente para tratar el SARS (síndrome respiratorio agudo grave) y el MERS (síndrome respiratorio de Oriente Medio) son candidatos potenciales para tratar la COVID-194,5.

\section{Tratamiento antiviral}

\section{Lopinavir/ritonavir}

Agente en combinación oral aprobado por la Administración de Drogas y Alimentos de los Estados Unidos (FDA) para tratar el virus de la inmunodeficiencia humana, demostró actividad in vitro frente a nuevos CoV mediante la inhibición de la proteasa 3-quimotripsina. Sin embargo no existen datos in vitro publicados para lopinavir/ritonavir (LPV/r) en relación con el SARS-CoV-2. Estudios clínicos en el SARS se asociaron con tasas reducidas de mortalidad e intubación, pero su naturaleza retrospectiva y observacional evitan conclusiones definitivas ${ }^{6,7}$. El régimen de dosificación de LPV/r más utilizado y estudiado para el tratamiento de la COVID-19 es de 400/100 mg dos veces al día hasta por 14 días. Dadas las interacciones farmacológicas significativas y las posibles reacciones adversas a estos medicamentos (náuseas y diarrea en 
Tabla 1. Resumen de farmacología para los tratamientos propuestos seleccionados para COVID-19

\begin{tabular}{|c|c|c|c|c|c|c|}
\hline Agente & Objetivo & $\begin{array}{l}\text { Dosis/administración } \\
\text { para adultos }\end{array}$ & Contraindicaciones & Toxicidad & $\begin{array}{l}\text { Interacciônes } \\
\text { farmacológicas } \\
\text { principalês }\end{array}$ & $\begin{array}{l}\text { Poblaciones } \\
\text { especiales }\end{array}$ \\
\hline $\begin{array}{l}\text { Fosfato de } \\
\text { cloroquina } \\
\left(\text { Aralen }^{\oplus}\right)\end{array}$ & $\begin{array}{l}\text { Bloqueo de la } \\
\text { entrada viral } \\
\text { mediante la } \\
\text { inhibición de la } \\
\text { glucosilación de los } \\
\text { receptores del } \\
\text { huésped, el } \\
\text { procesamiento } \\
\text { proteolítico y la } \\
\text { acidificación } \\
\text { endosómica. Efectos } \\
\text { inmunomoduladores } \\
\text { adicionales mediante } \\
\text { la inhibición de la } \\
\text { producción de } \\
\text { citocinas, la autofagia } \\
\text { y la actividad } \\
\text { lisosómica en las } \\
\text { células huésped }\end{array}$ & $\begin{array}{l}500 \text { mg por vía oral cada 12- } \\
24 \text { h × 5-10 d. Disponible como: } \\
\text { tabletas de } 250 \text { mg (sal); tabletas de } \\
500 \text { mg (sal); comprimidos de } 500 \mathrm{mg} \\
\text { de fosfato de cloroquina (sal) = } 300 \mathrm{mg} \\
\text { a base de cloroquina } \\
\text { Ajustes de dosis. Riñón: aclaramiento de } \\
\text { creatinina < } 10 \mathrm{ml} / \mathrm{min} \text { administra el } \\
50 \% \text { de la dosis. Hepático: no se } \\
\text { recomiendan ajustes de dosis en } \\
\text { insuficiencia hepática. Usar con } \\
\text { precaución } \\
\text { Administración: preferible evitar } \\
\text { aplastar. Si es necesario, se puede } \\
\text { triturar y mezclar con mermelada, yogur } \\
\text { pasteurizado o alimentos similares }\end{array}$ & $\begin{array}{l}\text { Hipersensibilidad a la } \\
\text { cloroquina, } \\
\text { compuestos de } \\
\text { 4-aminoquinolina o } \\
\text { cualquier } \\
\text { componente de la } \\
\text { formulación. Presencia } \\
\text { de cambios en la } \\
\text { retina o en el campo } \\
\text { visual de cualquier } \\
\text { etiología (a menos } \\
\text { que el beneficio } \\
\text { supere el riesgo) }\end{array}$ & $\begin{array}{l}\text { Frecuentes: calambres } \\
\text { abdominales, anorexia, } \\
\text { diarrea, náuseas, } \\
\text { vómitos. Mayores: } \\
\text { efectos } \\
\text { cardiovasculares } \\
\text { (incluida la } \\
\text { prolongación de QTc), } \\
\text { efectos hematológicos } \\
\text { (incluida la hemólisis } \\
\text { con deficiencia de } \\
\text { G6PD, el uso si el } \\
\text { beneficio supera los } \\
\text { riesgos), hipoglucemia, } \\
\text { toxicidad retiniana, } \\
\text { efectos } \\
\text { neuropsiquiátricos y } \\
\text { del sistema nervioso } \\
\text { central, reacciones } \\
\text { adversas } \\
\text { idiosincráticas }\end{array}$ & 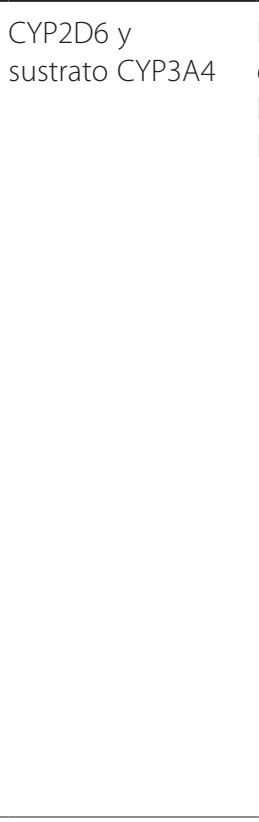 & $\begin{array}{l}\text { Puede usarse en } \\
\text { el embarazo si el } \\
\text { beneficio supera } \\
\text { los riesgos }\end{array}$ \\
\hline $\begin{array}{l}\text { Sulfato de } \\
\text { hidroxicloroquina } \\
\text { (Plaqueni }^{\oplus} \text { ) }\end{array}$ & $\begin{array}{l}\text { La hidroxicloroquina } \\
\text { comparte el mismo } \\
\text { mecanismo de } \\
\text { acción que la } \\
\text { cloroquina }\end{array}$ & $\begin{array}{l}400 \text { mg por vía oral cada } 12 \text { h × } 1 \text { d, } \\
\text { luego } 200 \text { mg por vía oral } \\
\text { cada } 12 \text { h × } 4 \text { d. Dosificación alternativa: } \\
400 \text { mg por vía oral al día } \times 5 \text { d, } 200 \text { mg } \\
\text { por vía oral } 3 \text { veces/día durante } 10 \text { días } \\
\text { Disponible como: tabletas de } 200 \text { mg de } \\
\text { sulfato de hidroxicloroquina } \\
\text { (sal) = } 155 \text { mg a base de } \\
\text { hidroxicloroquina. Ajustes de dosis: no } \\
\text { se recomiendan ajustes de dosis } \\
\text { hepáticos o renales. Usar con precaución } \\
\text { Administración: el fabricante no } \\
\text { recomienda tabletas trituradoras; sin } \\
\text { embargo, algunas fuentes sugieren que } \\
\text { las tabletas se pueden triturar y dispersar } \\
\text { con agua o mezclar en una solución oral }\end{array}$ & $\begin{array}{l}\text { Hipersensibilidad } \\
\text { conocida a la } \\
\text { hidroxicloroquina, } \\
\text { derivado de } \\
\text { 4-aminoquinolina, o } \\
\text { cualquier } \\
\text { componente de la } \\
\text { formulación }\end{array}$ & $\begin{array}{l}\text { Reacciones adversas a } \\
\text { los medicamentos } \\
\text { similares a la } \\
\text { cloroquina pero } \\
\text { menos frecuentes }\end{array}$ & 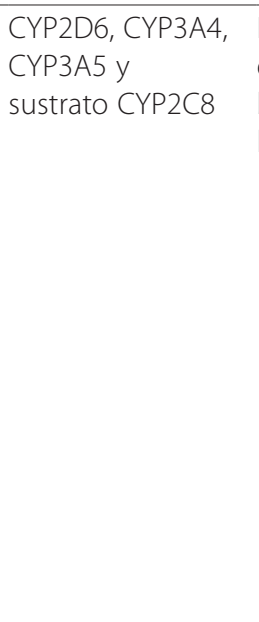 & $\begin{array}{l}\text { Puede usarse en } \\
\text { el embarazo si el } \\
\text { beneficio supera } \\
\text { los riesgos }\end{array}$ \\
\hline
\end{tabular}


Tabla 1. Resumen de farmacología para los tratamientos propuestos seleccionados para COVID-19 (continuación)

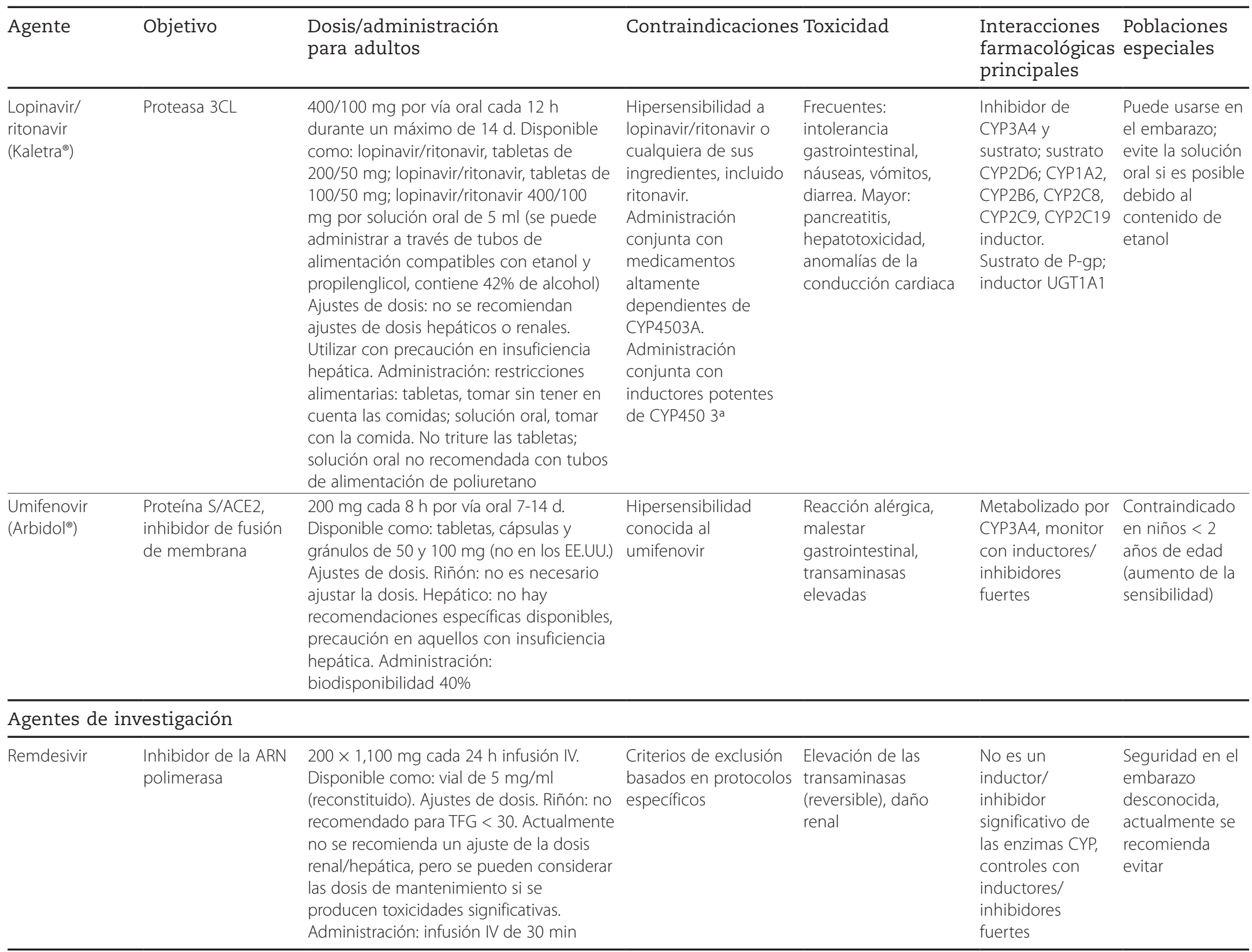


Tabla 1. Resumen de farmacología para los tratamientos propuestos seleccionados para COVID-19 (continuación)

\begin{tabular}{|c|c|c|c|c|c|c|}
\hline Agente & Objetivo & $\begin{array}{l}\text { Dosis/administración } \\
\text { para adultos }\end{array}$ & \multicolumn{2}{|c|}{ Contraindicaciones Toxicidad } & $\begin{array}{l}\text { Interaccioones } \\
\text { farmacológicas } \\
\text { principalềs }\end{array}$ & $\begin{array}{l}\text { Poblaciones } \\
\text { especiales }\end{array}$ \\
\hline Favipiravir & $\begin{array}{l}\text { Inhibidor del ARN } \\
\text { polimerasa }\end{array}$ & $\begin{array}{l}\text { Las dosis varían según la indicación, } \\
\text { datos limitados disponibles. Disponible } \\
\text { como: tableta de } 200 \text { mg (no en los } \\
\text { EE.UU.). Ajustes de dosis. Riñón: no se } \\
\text { recomienda ajuste de dosis, datos } \\
\text { limitados disponibles. Hepático: ajuste de } \\
\text { dosis considerado en Child-Pugh C, } \\
\text { aumento de exposiciones observadas en } \\
\text { Child-Pugh clase A-C. } \\
\text { Administración: la tableta se puede } \\
\text { triturar o mezclar con líquido, } \\
\text { biodisponibilidad > 95\% }\end{array}$ & $\begin{array}{l}\text { Criterios de exclusión } \\
\text { basados en protocolos } \\
\text { específicos }\end{array}$ & $\begin{array}{l}\text { Hiperuricemia, diarrea, } \\
\text { transaminasas } \\
\text { elevadas, reducción } \\
\text { del recuento de } \\
\text { neutrófilos }\end{array}$ & 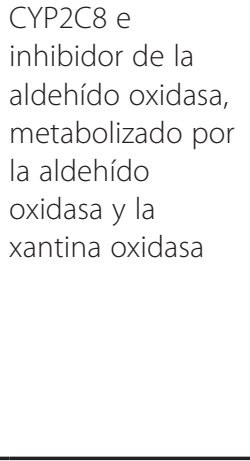 & $\begin{array}{l}\text { Contraindicado } \\
\text { durante el } \\
\text { embarazo, } \\
\text { metabolito } \\
\text { encontrado en } \\
\text { la leche } \\
\text { materna }\end{array}$ \\
\hline \multicolumn{7}{|c|}{ Terapias complementarias } \\
\hline $\begin{array}{l}\text { Tocilizumab } \\
\left(\text { Actemra }^{\oplus}\right)\end{array}$ & $\begin{array}{l}\text { Inhibición de IL-6: } \\
\text { reducción en la } \\
\text { tormenta de } \\
\text { citocinas }\end{array}$ & $\begin{array}{l}400 \mathrm{mg} \text { IV u } 8 \mathrm{mg} / \mathrm{kg} \times 1-2 \text { dosis. } \\
\text { Segunda dosis } 8-12 \text { h después de la } \\
\text { primera dosis si la respuesta es } \\
\text { inadecuada. Disponible como: inyección } \\
\text { de infusión IV: } 80 \mathrm{mg} / 4 \mathrm{ml}(20 \mathrm{mg} / \mathrm{ml}) ; \\
200 \mathrm{mg} / 10 \mathrm{ml}(20 \mathrm{mg} / \mathrm{ml}) ; 400 \mathrm{mg} / 20 \mathrm{ml} \\
\text { ( } 20 \mathrm{mg} / \mathrm{ml} \text { ) en viales de dosis única para } \\
\text { una dilución adicional antes de la } \\
\text { infusión intravenosa. } \\
\text { Ajustes de dosis. Riñón: no se } \\
\text { recomiendan ajustes de dosis en } \\
\text { insuficiencia renal leve o moderada. No } \\
\text { estudiado en pacientes con discapacidad } \\
\text { grave. Hepático: no se recomiendan } \\
\text { ajustes de dosis (no estudiados); iniciar } \\
\text { basado en el beneficio. Administración: } \\
\text { infundir durante } 60 \text { minutos, no se debe } \\
\text { infundir concomitantemente en la misma } \\
\text { línea IV con otros fármacos }\end{array}$ & $\begin{array}{l}\text { Hipersensibilidad } \\
\text { conocida al } \\
\text { tocilizumab o } \\
\text { cualquiera de los } \\
\text { componentes de la } \\
\text { formulación. } \\
\text { Precaución en } \\
\text { pacientes con } \\
\text { neutropenia }(<500 \\
\text { células/ } \mu \text { l) o } \\
\text { trombocitopenia } \\
(<50,000 / \mu l)\end{array}$ & $\begin{array}{l}\text { Frecuentes: aumento } \\
\text { de las infecciones del } \\
\text { tracto respiratorio } \\
\text { superior (incluida la } \\
\text { tuberculosis), } \\
\text { nasofaringitis, dolor } \\
\text { de cabeza, } \\
\text { hipertensión, } \\
\text { aumento de AST, } \\
\text { reacciones } \\
\text { relacionadas con la } \\
\text { perfusión. } \\
\text { Mayor: efectos } \\
\text { hematológicos, } \\
\text { infecciones, } \\
\text { hepatotoxicidad, } \\
\text { perforaciones } \\
\text { gastrointestinales, } \\
\text { reacciones de } \\
\text { hipersensibilidad }\end{array}$ & 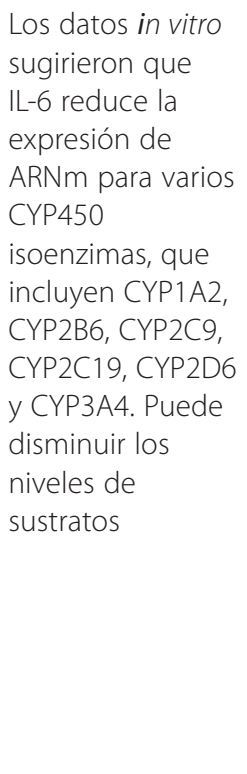 & $\begin{array}{l}\text { Seguridad en el } \\
\text { embarazo } \\
\text { desconocida; } \\
\text { puede causar } \\
\text { daño al feto }\end{array}$ \\
\hline
\end{tabular}

ACE2: enzima convertidora de angiotensina II; AST: aspartato aminotransferasa; 3CL: similar a 3-quimotripsina; COVID-19: enfermedad por coronavirus 2019; CYP: citocromo P450; G6PD: છ̈lucosa-6-fosfato-

deshidrogenasa; TFG: tasa de filtración glomerular; IV: intravenosa; P-gp: P-glucoproteína; UGT1A1: UDP glucuronosiltransferasa familia 1 miembro A1; IL: interleucina.

Adaptada de Sanders, et al., $2020^{5}$. 
un 28\%, hepatotoxicidad en el 2 al 10\%, transaminasas elevadas en el 20 al $30 \%$ y pancreatitis en el $2.1 \%$ ), se requiere una revisión cuidadosa de los medicamentos concomitantes y el monitoreo de su uso ${ }^{5,8-10}$. Una revisión sistemática reciente mostró que el efecto anti-CoV del LPV/r se observó principalmente en su aplicación temprana (desde el inicio de los síntomas hasta los 12 días posteriores al inicio de los síntomas)para reducir la mortalidad del paciente, adicionando el uso de glucocorticoides (40 a $80 \mathrm{mg}$ de metilprednisolona por día, la dosis diaria total no debe exceder los $2 \mathrm{mg} / \mathrm{kg}$, de 3 a 5 días como máxi$\mathrm{mo}^{3}$, sin embargo, si se pierde la ventana de tratamiento temprano, no habrá un efecto significativo en su aplicación tardía. La efectividad del uso combinado de antivirales sigue siendo controvertida ${ }^{11,12}$.

\section{Ribavirina}

Análogo de la guanina, inhibe la ARN polimerasa dependiente de ARN viral. Su actividad contra otros CoV lo hace candidato para el tratamiento de la COVID-19. Sin embargo, su actividad in vitro contra el SARS-CoV es limitada y requiere altas concentraciones para inhibir la replicación viral. La ribavirina causa toxicidad hematológica grave dependiente de la dosis ${ }^{5,13}$.

\section{Otros antivirales}

El oseltamivir, un inhibidor de la neuraminidasa aprobado para el tratamiento del virus de la influenza tipo $A$ y la $B$, no tiene ningún papel en el manejo de la COVID-19 una vez que la influenza ha sido excluida.

El umifenovir (también conocido como Arbidol ${ }^{\circledR}$ ) es un agente antiviral que cuenta con un mecanismo de acción único dirigido a la interacción de la proteína S/enzima convertidora de angiotensina II (ACE2) e inhibe la fusión de la membrana de la envoltura viral. Este agente está aprobado actualmente en Rusia y China para el tratamiento y profilaxis de la influenza, y es de creciente interés para el tratamiento de la COVID-19 con base en datos in vitro que sugieren actividad contra el SARS. Los datos de estudios observacionales realizados en China no pueden establecer la eficacia del umifenovir para la COVID-195.

\section{Terapia antipalúdica, cloroquina e hidroxicloroquina}

Estos fármacos tienen una larga historia en la prevención y el tratamiento de la malaria y el tratamiento de enfermedades inflamatorias crónicas, como el lupus eritematoso sistémico y la artritis reumatoide. Su mecanismo de acción parece bloquear la entrada viral a las células mediante la inhibición de la glucosilación de los receptores del huésped, el procesamiento proteolítico y la acidificación del endosoma. Estos agentes también tienen efectos inmunomoduladores mediante la atenuación de la producción de citocinas y la inhibición de la autofagia y la actividad lisosómica en las células huésped ${ }^{5}$. La hidroxicloroquina (200 mg, por vía oral cada 8 h) tiene actividad in vitro más baja para el SARS-CoV-2 en comparación con la cloroquina (500 mg por vía oral una o dos veces al día), sin embargo, ambos agentes pueden causar efectos adversos raros y graves $(<10 \%)$, incluida la prolongación del QTc, la hipoglucemia, efectos neuropsiquiátricos y la retinopatía, además del potencial de arritmias, especialmente en pacientes críticos y aquellos que toman medicamentos concomitantes para prolongar el intervalo QT, como azitromicina y fluoroquinolonas ${ }^{14}$.

\section{Terapia antibiótica}

Se ha reportado que el uso ciego o inapropiado de medicamentos antibacterianos, especialmente la combinación de medicamentos antibacterianos de amplio espectro, repercute negativamente en la evolución del paciente infectado con el SARS-CoV-2. Debe realizarse vigilancia bacteriológica y administrarse medicamentos antibacterianos apropiados de inmediato solo cuando se produce una infección bacteriana secundaria. De acuerdo con las manifestaciones clínicas de los pacientes afectados por COVID-19, si no se puede descartar la infección bacteriana que lo acompaña, los pacientes con enfermedad leve pueden tomar medicamentos antibacterianos contra la neumonía adquirida en la comunidad, como amoxicilina, azitromicina o fluoroquinolonas. El tratamiento antibacteriano empírico en pacientes con enfermedad severa debe cubrir todos los patógenos posibles ${ }^{12}$. 


\section{Terapia con corticosteroides}

El uso de corticosteroides para el síndrome de dificultad respiratoria aguda (SDRA) grave es controvertido; por lo tanto, el uso sistémico de glucocorticoides debe ser cauteloso. La metilprednisolona se puede usar según sea apropiado para pacientes con progresión rápida de la enfermedad o enfermedad grave. Según la gravedad de la enfermedad, se pueden considerar de 40 a $80 \mathrm{mg}$ de metilprednisolona por día, y la dosis diaria total no debe exceder los $2 \mathrm{mg} / \mathrm{kg}$. Las investigaciones relacionadas con el manejo del SARS mostraron que el uso oportuno de la presión positiva continua no invasiva de las vías respiratorias y los corticosteroides es una estrategia efectiva para mejorar significativamente los síntomas clínicos de pacientes con SARS, reducir el grado de progresión de la enfermedad y acelerar la absorción de las lesiones pulmonares ${ }^{15,16}$.

\section{Agentes misceláneos}

El interferón a y $\beta$ se ha estudiado para nuevos CoV, conocidos por su actividad contra el interferón $\beta$. Las guías chinas actuales enumeran los interferones como una alternativa para la terapia combinada. Varios otros agentes inmunomoduladores (baricitinib, imatinib, dasatinib y ciclosporina) utilizados tradicionalmente para indicaciones no infecciosas poseen mecanismos compatibles para inhibir el SARS-CoV-2, sin embargo, no existen datos en animales o humanos para recomendar su uso para la COVID-195,17.

\section{Agentes ACE2 y ARB}

El SARS-CoV-2 usa el receptor ACE2 para ingresar a la célula huésped. Este descubrimiento ha estimulado las discusiones sobre si los inhibidores de la ACE2 y/o los bloqueadores de los receptores de angiotensina (ARB) pueden potencialmente tratar la COVID-19 o, por el contrario, empeorar la enfermedad ${ }^{5}$. Algunos estudios sugieren que la expresión de la ACE2 aumenta en la diabetes, lo que facilitaría la infección por SARS-CoV- $2^{18}$. Ante esta posibilidad, se realizó recientemente una búsqueda en PubMed, en la cual no se encontró ninguna evidencia consistente que sugiera el desuso de dichos fármacos; además se corroboró la seguridad de los antihipertensivos bloqueadores de los canales de calcio, ya que no aumentan la expresión o actividad de la ACE2, por lo tanto, estos podrían ser un tratamiento alternativo adecuado en aquellos pacientes en los que esté justificado el beneficio del cambio de la terapia antihipertensiva convencional, de acuerdo con el estado de enfermedad del paciente y las comorbilidades relacionadas 3,19 . Existen datos contradictorios in vitro para determinar si estos agentes tienen un efecto negativo o protector en pacientes hospitalizados con COVID-195.

\section{Remdesivir}

Formalmente conocido como GS-5734, es un profármaco de monofosfato que sufre metabolismo a un análogo activo de nucleósido trifosfato de Cadenosina, con actividad contra virus ARN, como Coronaviridae y Flaviviridae. Este agente mostró eficacia en el brote de Ébola. Actualmente, el remdesivir es una terapia potencial prometedora para la COVID-19 debido a su potente actividad in vitro de amplio espectro contra varios nuevos CoV, incluido el SARS-CoV- $2^{20}$. La seguridad y la farmacocinética del remdesivir se evaluaron en ensayos clínicos de fase 1 de dosis única y múltiple. La dosis actual bajo investigación es una dosis de carga única de 200 $\mathrm{mg}$, seguida de una infusión diaria de $100 \mathrm{mg}$. No se recomiendan ajustes hepáticos o renales en este momento, pero no se recomienda el inicio en pacientes con una tasa de filtración glomerular estimada inferior a $30 \mathrm{ml} / \mathrm{min}$. Se están realizando ensayos clínicos para evaluar la seguridad y la actividad antiviral del remdesivir en pacientes con COVID-19 leve, moderada o grave ${ }^{5,21}$.

\section{Anticitocinas o los inmunomoduladores}

Los anticuerpos monoclonales dirigidos contra las citocinas inflamatorias clave y otros aspectos de la respuesta inmunitaria innata representan otra clase potencial de terapias complementarias para la COVID-19. La razón de su uso es que la fisiopatología subyacente del daño orgánico significativo en los 
pulmones y otros órganos es causada por una respuesta inmunitaria amplificada y la liberación de citocinas, la interleucina 6 (IL-6) parece ser un factor clave de esta inflamación desregulada basada en series de casos tempranos de China, por lo tanto, los anticuerpos monoclonales contra la IL-6 (tocilizumab, sarilumab) podrían en teoría amortiguar este proceso y mejorar los resultados clínicos. Otros anticuerpos monoclonales o agentes inmunomoduladores en ensayos clínicos en China incluyen el bevacizumab (medicación antifactor de crecimiento endotelial vascular; NCT04275414), el fingolimod (inmunomodulador aprobado para esclerosis múltiple; NCT04280588) y el eculizumab (anticuerpo inhibidor del complemento terminal;NCT04288713) ${ }^{5}$.

\section{Inmunoglobulina}

Otra terapia adyuvante potencial para la COVID-19 es el uso de inmunoglobulinas plasmáticas o hiperinmunes. La justificación de este tratamiento es que los anticuerpos de pacientes recuperados pueden ayudar tanto con el virus libre como con el aclaramiento inmunitario de las células infectadas. Los informes o protocolos anecdóticos para plasma convaleciente han sido reportados como terapia de rescate en el SARS y el MERS 5 .

La estrategia a largo plazo más efectiva para la prevención de brotes futuros de este virus sería el desarrollo de una vacuna que proporcione inmunidad protectora; sin embargo, se requeriría un mínimo de 12 a 18 meses antes del despliegue generalizado de la vacuna ${ }^{5}$, además de general evidencia científica proveniente de ensayos clínicos (Tabla 2).

\section{INFECCIÓN POR SARS-COV-2} Y DIABETES

Los pacientes con diabetes tienen mayor riesgo de morbilidad y mortalidad con respecto a la infección por SARS-CoV-2. La hiperglucemia crónica afecta negativamente la función inmunitaria, aumentando por ende el riesgo de morbilidad y mortalidad debido a cualquier infección, incluida la COVID-19, además se encuentra asociada a complicaciones orgánicas múltiples. La mortalidad en pacientes con diabetes y COVID-19 en China es de alrededor del 7.3\%1. La diabetes estuvo presente en el $42.3 \%$ de las 26 muertes por COVID-19 en Wuhan, China.

Un informe de 72,314 casos de COVID-19 publicado por el Centro Chino para el Control y la Prevención de Enfermedades mostro una mayor mortalidad en personas con diabetes ( $2.3 \%$ en población general y $7.3 \%$ en pacientes con diabetes) ${ }^{22}$.

Las recomendaciones para las personas que viven con diabetes durante la pandemia por COVID-191,4 son las siguientes:

- Estricta adherencia a las medidas preventivas generales.

- Distanciamiento social.

- Indicar a los pacientes que continúen tomando sus medicamentos recetados cotidianamente.

- Indicar a los pacientes que adquieran medicamentos y suministros (30 días de insulina $\mathrm{y} / \mathrm{u}$ otros medicamentos para la diabetes) cuando se declare estado de emergencia sanitaria.

- Buen control y monitoreo de la glucosa, por medio de llamadas telefónicas, videoconferencias o información digital.

- Los pacientes con diabetes tipo 1 deben medir la glucosa en sangre y las cetonas urinarias con

- Los pacientes con diabetes tipo 1 deben medir la glucosa en sangre y las cetonas urinarias con frecuencia si se presenta fiebre e hiperglucemia, ya que pueden ser necesarios cambios frecuentes en la dosis y bolos correccionales para mantener la normoglucemia 22 .

- Solo personal capacitado y protegido ante la exposición deberá asesorar presencialmente a las personas con diabetes sobre las medidas específicas relacionadas con el manejo de su enfermedad en caso de infección por SARS-CoV-2.

- Evitar la desnutrición con dietas o medidas adyuvantes si esta clínicamente indicado.

Las personas con diabetes con COVID-19 pueden experimentar un deterioro del control glucémico 
Tabla 2. Resumen del tratamiento y resultados clínicos de las primeras series clínicas de COVID-19

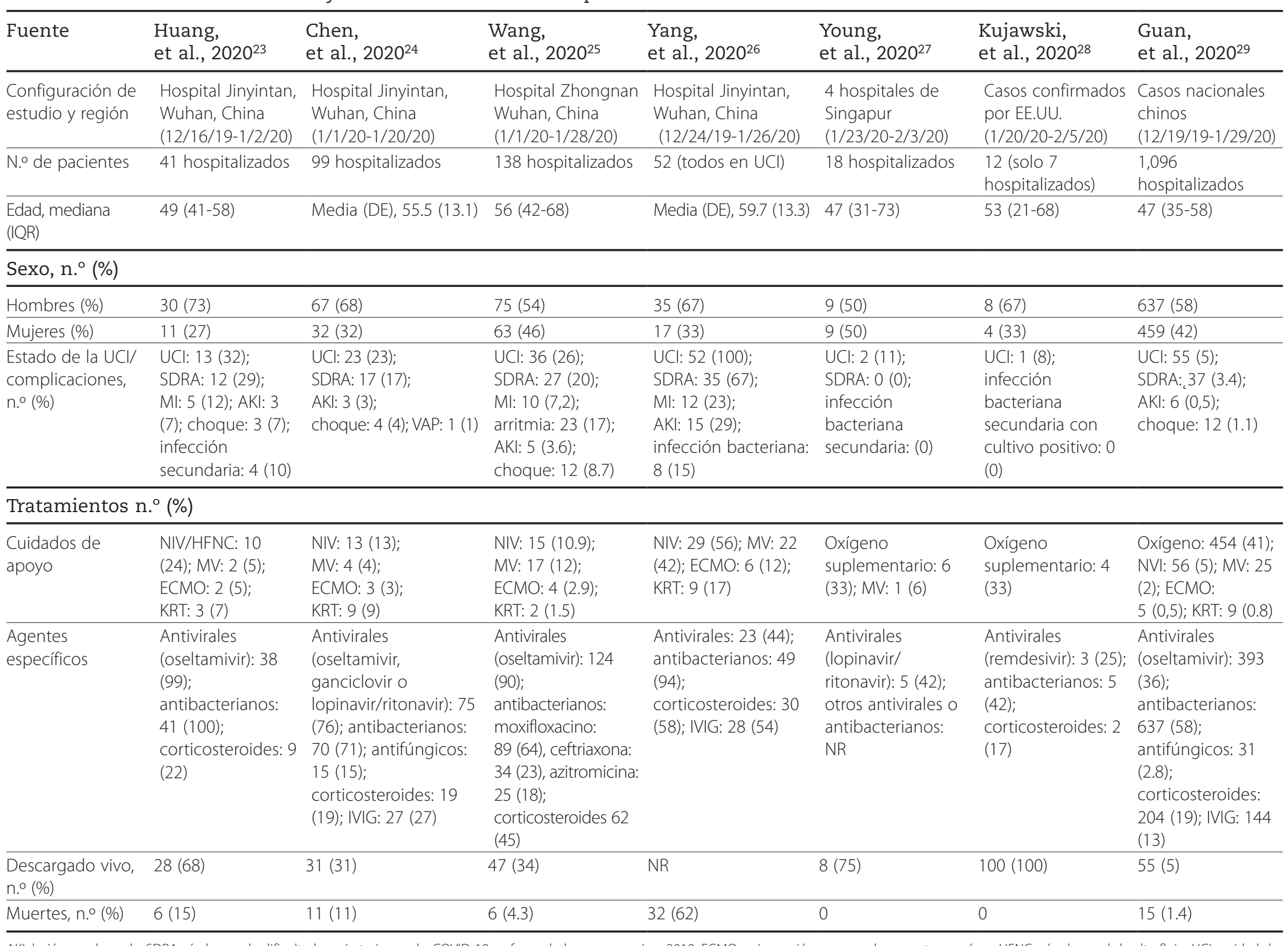

AKI: lesión renal aguda; SDRA: síndrome de dificultad respiratoria aguda; COVID-19: enfermedad por coronavirus 2019; ECMO: oxigenación por membrana extracorpórea; HFNC: cánula năsal de alto flujo; UCI: unidad de cuidados intensivos; IQR: rango intercuartílico; IVIG: inmunoglobulinas intravenosas; MI: infarto de miocardio; MV: ventilación mecánica invasiva; KRT: terapia de reemplazo renal; NIV: ventilación no invasiva; NR: no

informado; VAP: neumonía asociada al ventilador. 
durante la enfermedad, como en cualquier otro episodio infeccioso, por lo tanto es de suma importancia la comunicación médico-paciente ante signos y síntomas de COVID-19, para poder generar un manejo adecuado, pronto y optimo del equipo multidisciplinario'.

\section{COVID-19 Y OBESIDAD}

Actualmente, según la experiencia de algunos hospitales en España, se ha observado que los casos de jóvenes en los que hay obesidad severa pueden evolucionar hacia una alveolitis destructiva con insuficiencia respiratoria y muerte. No hay una explicación actual para esta presentación clínica, aunque es bien sabido que la obesidad severa está asociada al síndrome de apnea del sueño, así como a la disfunción del factor surfactante, que puede contribuir a un peor escenario en el caso de la infección por SARS-CoV-2 ${ }^{1}$.

RECOMENDACIONES PARA ENDOCRINÓLOGOS QUE ATIENDAN

\section{PACIENTES CON COVID-19}

- Protéjase adecuadamente con el equipo de protección personal (EPP) completo y solicite la prueba de la COVID-19 si está expuesto.

- Evite citas rutinarias innecesarias en persona.

- Establezca servicios de consulta en línea/correo electrónico/teléfono, etc.

- Monitoree de cerca el control glucémico en pacientes con diabetes.

- Recomiende a las personas con diabetes una estricta adherencia a las medidas preventivas generales.

- Asesore a las personas con diabetes sobre medidas específicas relacionadas con el manejo de su enfermedad en caso de infección por SARSCoV-2.
- Asesore a las personas con diabetes, especialmente si tienen más de 65 años y son obesas, sobre las derivaciones para el tratamiento en caso de sospecha de infección por SARS-CoV-2.

- Evite la desnutrición con medidas dietéticas o complementarias si esta clínicamente indicado.

- Monitoree de cerca las condiciones clínicas de los pacientes con diabetes, obesidad y problemas endocrinos agregados a la COVID-19.

PLANES DE TRATAMIENTO DURANTE LA HOSPITALIZACIÓN PARA LOS CASOS CONFIRMADOS DE INFECCIÓN POR

\section{SARS-COV-2}

En la actualidad, en ausencia de terapia preventiva para la infección por SARS-CoV-2, la piedra angular de la atención para pacientes con COVID-19 sigue siendo la atención de apoyo, que va desde el tratamiento ambulatorio sintomático hasta el tratamiento intensivo completo ${ }^{5}$.

El manejo del paciente hospitalizado por COVID-19 incluye las siguientes indicaciones ${ }^{1,3,4}$ :

- El paciente debe descansar en cama, vigilar sus signos vitales (frecuencia cardiaca, saturación de oxígeno del pulso, frecuencia respiratoria, presión arterial por monitorización continua) y garantizar tratamiento de apoyo para mantener un equilibrio hidroelectrolítico y de energía.

- El paciente debe ser monitoreado por análisis sanguíneos habituales, proteína $\mathrm{C}$ reactiva, procalcitonina, función de los órganos (enzimas hepáticas, bilirrubina, enzimas miocárdicas, creatinina, nitrógeno ureico, volumen de orina, etc.), función de coagulación, análisis de gases en sangre arterial e imágenes de tórax de ser posible cada $24 \mathrm{~h}^{3}$.

- El paciente debe recibir una terapia de oxígeno eficaz, que incluye: catéter nasal, mascarilla de oxígeno, terapia de oxígeno nasal de alto flujo (HFNO), ventilación no invasiva (NIV) o ventilación mecánica invasiva (VMI); las terapias de bajo flujo (puntas nasales, mascarillas simples o mascarillas 
con reservorio) no produjeron aumento en el riesgo de contaminación por aerosoles, sí en cambio las terapias de alto flujo, que tienen el potencial riesgo de propagación de aerosoles infecciosos, por lo cual deberán tomarse las precauciones estándares al administrarlas ${ }^{30}$.

- Cuando la temperatura corporal del paciente es superior a $38.5^{\circ} \mathrm{C}$, el paracetamol se puede usar como antipirético (oral, $650 \mathrm{mg}$ por vez, cada 6-8 h en fiebre continua, pero no más de 4 veces en $24 \mathrm{~h}$ ), y la temperatura por debajo de $38^{\circ} \mathrm{C}$ es aceptable y no amerita tratamiento farmacológico.

- Los pacientes deberán ser evaluados para determinar el riesgo nutricional a su ingreso según la puntuación sugerida en cada centro (puntuación de detección de riesgo nutricional 2002 [NRS 2002] $)^{31}$. El plan recomendado para pacientes con diferentes puntuaciones de riesgo nutricional es el siguiente:

- Si la puntuación total es $<3$ puntos, se recomienda comer alimentos ricos en proteínas (como huevos, pescado, carne magra, productos lácteos) y dietas que contengan carbohidratos. La ingesta ideal de energía es de $25-30 \mathrm{kcal} / \mathrm{kg} / \mathrm{d}$, proteínas $1.5 \mathrm{~g} / \mathrm{kg} / \mathrm{d}$.

- Si la puntuación total es $\geq 3$ puntos, el paciente debe recibir apoyo nutricional lo antes posible. Se recomienda aumentar la ingesta de proteínas mediante un suplemento de nutrición oral, 2-3 veces/día ( $\geq 18 \mathrm{~g}$ de proteína/tiempo). Para alcanzar la cantidad de $18 \mathrm{~g}$ de proteína/tiempo, se puede agregar proteína en polvo sobre la base de preparaciones estándar de proteínas enteras. La nutrición enteral debe colocarse cuando el paciente no puede ingerir nutrición suplementaria oral.

- Se deberá reducir la incidencia de úlceras gástricas por estrés y sangrado gastrointestinal. Se sugiere el uso de antagonistas del receptor de histamina 2 (p. ej., cimetidina $50 \mathrm{mg} / \mathrm{h}$ IV, infusión continua o 300 mg cada 6 h por sonda nasogástrica [SNG], dosis diaria máxima de 2,400 mg; famotidina $20 \mathrm{mg}$ cada $12 \mathrm{~h}$ por SNG) o inhibidores de la bomba de protones (p. ej., esomeprazol, pantoprazol u omeprazol $40 \mathrm{mg}$ IV cada $24 \mathrm{~h})^{32}$ en pacientes con factores de riesgo de hemorragia gastrointestinal. Los factores de riesgo para el sangrado gastrointestinal incluyen: ventilación mecánica $\geq 48 \mathrm{~h}$, disfunción de la coagulación, terapia de reemplazo renal, enfermedad hepática, diversas complicaciones y una mayor puntuación de insuficiencia orgánica.

- Se deberá reducir la secreción de las glándulas pulmonares y mejorar la función respiratoria. Para los pacientes con disnea, tos, sibilancias y síndrome de dificultad respiratoria se recomienda usar fármacos selectivos de receptores anticolinérgicos $M 1, M 3$ (darifenacina 10 o $20 \mathrm{mg} /$ día por SNG o hioscina $0.6 \mathrm{mg} /$ día por SNG) para reducir la secreción, relajar el músculo liso de las vías respiratorias, aliviar el espasmo y mejorar la ventilación pulmonar ${ }^{33}$.

- Se deberá reducir la incidencia de embolia venosa. Se sugiere evaluar el riesgo de embolia venosa en pacientes de alto riesgo, y en caso necesario usar heparina o heparina de bajo peso molecular.

\section{TRATAMIENTO DE PACIENTES CON ENFERMEDAD SEVERA}

\section{Tratamientos de la insuficiencia respiratoria hipoxémica y del SDRA}

EI SDRA es un estado de insuficiencia respiratoria hipóxica aguda grave causado por el aumento de la permeabilidad capilar pulmonar y el daño de las células epiteliales alveolares. Se puede dividir según la definición de Berlín para el SDRA:

- Tiempo de inicio. Síntomas respiratorios desarrollados/agravados dentro de una semana después del daño clínicamente conocido.

- Causas del edema pulmonar. La insuficiencia respiratoria no puede explicarse completamente por insuficiencia cardiaca o sobrecarga de líquidos. Se necesita una evaluación objetiva (como la ecocardiografía) para eliminar la posibilidad de edema pulmonar hidrostático si no hay otro factor de riesgo.

- Anormalidad en imagen. La disminución de la transparencia de dos pulmones no puede explicarse 
completamente por derrame pleural, atelectasia o nódulos.

- Hipoxemia. Que puede ser:

- Leve: presión arterial de oxígeno/fracción inspiratoria de oxígeno $\left(\mathrm{PaO}_{2} / \mathrm{FiO}_{2}\right)$ 201-300 mmHg, presión positiva al final de la espiración (PEEP) o presión positiva continua en la vía aérea (CPAP) $\geq 5$ $\mathrm{CmH}_{2} \mathrm{O}$.

- Moderada: $\mathrm{PaO}_{2} / \mathrm{FiO}_{2}$ 101-200 mmHg, PEEP $\geq 5 \mathrm{cmH}_{2} \mathrm{O}$.

- O severa: $\mathrm{PaO}_{2} / \mathrm{FiO}_{2} \leq 100 \mathrm{mmHg}$, PEEP $\geq 10 \mathrm{cmH}_{2} \mathrm{O}^{5}$.

El objetivo del tratamiento en este grupo de pacientes es mejorar los síntomas y las enfermedades subyacentes, prevenir activamente las posibles complicaciones e infecciones secundarias, además de proporcionar medidas oportunas para apoyar la función orgánica ${ }^{3,34}$.

Se sugieren estos tipos de apoyo ventilatorio ${ }^{3}$ :

- Oxigenoterapia a través de una máscara facial y una bolsa de reserva (flujo de gas de 10 a $15 \mathrm{l} / \mathrm{min}$, $\mathrm{FiO}_{2}$ 0.60-0.95).

- HFNO en pacientes polipneicos para mantener la saturación de oxígeno capilar periférica por encima del 93\%. Las medidas incluyen: flujo de gas inicialmente de $30-40 \mathrm{l} / \mathrm{min}$ y la concentración de oxígeno $50-60 \%$, lo cual es bien tolerado. El HFNO puede reducir la posibilidad de intubación traqueal. Los pacientes con hipercapnia (como exacerbación de enfermedad pulmonar obstructiva, edema pulmonar cardiogénico), inestabilidad hemodinámica, falla multiorgánica o estado mental anormal no deben recibir HFNO.

- La NIV proporciona un cierto efecto de ventilación con presión positiva a través de una máscara cerrada. El HFNO combinado con la NIV (1-2 h) puede ser útil para reducir el consumo de energía respiratoria y mejorar la oxigenación. Los pacientes con inestabilidad hemodinámica, insuficiencia orgánica múltiple o estado mental anormal no deben recibir NIV.

- VMI. Se sugiere en caso de SDRA si el paciente se deteriora gravemente. La estrategia de ventilación pulmonar protectora en la VMI se caracteriza por: volumen corriente bajo $(4-6 \mathrm{ml} / \mathrm{kg})$, presión de meseta baja $\left(<30 \mathrm{cmH}_{2} \mathrm{O}\right)$ y PEEP no mayor a $10 \mathrm{cmH}_{2} \mathrm{O}$.

La pandemia de la enfermedad causada por el SARS-CoV-2 presenta un desafío sin precedentes para identificar medicamentos efectivos para la prevención y el tratamiento. El conocimiento se expande rápidamente con los datos clínicos generados por la gran cantidad de personas infectadas y la virología del SARS-CoV-2, lo que proporciona un número significativo de objetivos farmacológicos potenciales; sin embargo, actualmente no hay evidencia de ensayos clínicos aleatorizados de que cualquier terapia potencial mejore los resultados en pacientes hospitalizados con COVID-19, tampoco existen datos de ensayos clínicos que respalden alguna terapia profiláctica ${ }^{5}$.

\section{RETOS DEL ENDOCRINÓLOGO ANTE} LOS NUEVOS TRATAMIENTOS

Los efectos secundarios de los diversos grupos de fármacos que se están utilizando actualmente para la COVID-19 no son del todo conocidos.

- Antirretrovirales. Los efectos secundarios metabólicos del uso de antirretrovirales como LPV/r son principalmente: hipercolesterolemia, hiperglucemia, resistencia a la insulina, hipertrigliceridemia (HTG), aterosclerosis asociada a HTG y pancreatitis asociada o no a HTG: Por lo tanto, algunas recomendaciones al usar LPV/r en pacientes con COVID-19 deberían ser: toma y evaluación de laboratorios (glucosa, triglicéridos y colesterol), al ingreso hospitalario del paciente o al menos antes del inicio del tratamiento con LPV/r y al término de su uso. Debido a que muchos casos de pancreatitis son subclínicos $y$, por lo tanto, pueden pasar desapercibidos, también puede ser útil evaluar la s-amilasa y la s-lipasa mensualmente ${ }^{35}$.

- Glucocorticoides. Aunque se prescriben ampliamente por sus propiedades antiinflamatorias e inmunosupresoras, tienen varios efectos secundarios metabólicos comunes que incluyen hipertensión, osteoporosis y diabetes. La tendencia de los 
pacientes a desarrollar una nueva hiperglucemia en el contexto del inicio de la terapia con glucocorticoides a menudo no se anticipa. En los ancianos, sin un seguimiento cercano o monitoreo de los niveles de glucosa en sangre existe el riesgo de precipitar estados hiperosmolares hiperglucémicos, lo que podría agravar aún más el cuadro ${ }^{36}$.

- Antipalúdicos, como la cloroquina y su derivado la hidroxicloroquina. Se ha demostrado que algunos pacientes con diabetes mellitus y en tratamiento con antipalúdicos recuperan la actividad transitoria de las células beta, que dura en la mayoría de los casos unas pocas semanas, meses o, raramente, años. En los pacientes con diabetes mellitus y que todavía cuentan con algo de actividad de células beta presente, podrían precipitar una hipoglucemia, que se asocia con una mayor mortalidad en sujetos hospitalizados con neumonía (independientemente de si tienen diabetes). Dichos pacientes deben ser monitoreados de cerca para detectar hipoglucemia y, si es necesario, la dosis de insulina deberá ajustarse, incluso después de la recuperación ${ }^{37,38}$. La base de este efecto hipoglucemiante es multifactorial e incluye un aclaramiento de insulina reducido, una mayor sensibilidad a la insulina y una mayor liberación de insulina pancreática ${ }^{39,40}$.

\section{CONCLUSIONES}

El reto ante la pandemia por COVID-19 es mayúsculo para la medicina actual. El endocrinólogo se enfrenta a un escenario sombrío, donde la seguridad y protección personal en el momento de la atención médica es el punto medular. La información actualizada y fiable mejorará la conducta terapéutica en estos pacientes, ayudando así a optimizar las condiciones clínicas preexistentes, como las enfermedades crónicas y metabólicas (p. ej., diabetes, hipertensión, obesidad, etc.), que implican un alto riesgo tanto de complicaciones como de letalidad.

Muchos fármacos se han probado recientemente en el tratamiento para la COVID-19. Todos estos fármacos tienen indicaciones distintas y precisas de tratamiento.
Numerosos informes han demostrado efectividad experimental in vitro e in vivo en el tratamiento de la COVID-19; dicha efectividad, así como los efectos secundarios y adversos de estos nuevos usos, no han sido evaluados completamente, por lo cual es de suma importancia investigar el impacto que tendrán estos fármacos en los pacientes a corto, mediano y largo plazo.

\section{FINANCIAMIENTO}

La presente investigación no ha recibido ayudas específicas provenientes de agencias del sector público, sector comercial o entidades sin ánimo de lucro.

CONFLICTO DE INTERESES

Los autores declaran no tener conflicto de intereses alguno.

RESPONSABILIDADES ÉTICAS

Protección de personas y animales. Los autores declaran que para esta investigación no se han realizado experimentos en seres humanos ni en animales.

Confidencialidad de los datos. Los autores declaran que en este artículo no aparecen datos de pacientes.

Derecho a la privacidad y consentimiento informado. Los autores declaran que en este artículo no aparecen datos de pacientes.

\section{BIBLIOGRAFÍA}

1. Puig-Domingo $M$, Marazuela $M$, Giustina A. COVID-19 and endocrine diseases. A statement from the European Society of Endocrinology. Endocrine. 2020;68:2-5.

2. Curley C. People with diabetes may have higher risk for COVID-19: Here's what to do [Internet]. Healthline [fecha de publicación: 19 de marzo de 
2020]. Disponible en: https://www.healthline.com/health-news/peoplewith-diabetes-risk-healthcare-covid19

3. Jin YH, Cai L, Cheng ZS, Cheng H, Deng T, Fan YP, et al. A rapid advice guideline for the diagnosis and treatment of 2019 novel coronavirus (2019-nCoV) infected pneumonia (standard version). Mil Med Res. 2020;7(1):4.

4. AACE Position Statement: Coronavirus (COVID-19) and people with diabetes (Updated March 18, 2020) [Internet]. American Association of Clinical Endocrinologists; 18 de marzo de 2020. Disponible en: https:// www.aace.com/recent-news-and-updates/aace-position-statement-coronavirus-covid-19-and-people-diabetes-updated

5. Sanders JM, Monogue ML, Jodlowski TZ, Cutrell JB. Pharmacologic treatments for coronavirus disease 2019 (COVID-19) A review. JAMA. 2020 Apr 13. doi: 10.1001/jama.2020.6019. Online ahead of print.

6. Yao TT, Qian JD, ZhuWY,Wang Y,Wang GQ. A systematic review of lopinavir therapy for SARS coronavirus and MERS coronavirus-A posible reference for coronavirus disease-19 treatment option. J Med Virol. 2020;92(6):556-63.

7. Chan KS, Lai ST, Chu CM, Tsui E, Tam CY, Wong MM, et al. Treatment of severe acute respiratory syndrome with lopinavir/ritonavir: a multicentre retrospective matched cohort study. Hong Kong Med J. 2003;9(6):399-406.

8. World Health Organization. WHO handbook for guideline development. $2^{\text {nd }}$ ed [Internet]. World Health Organization; 2014. Disponible en: https://apps.who.int/iris/handle/10665/145714

9. Brigitte $M$, Arti B, Stephan A, Judith A, Bruno R, Christoph W, et al. MERSCOV disease associated ARDS -a case report. Crit Care Med. 2015;43(12):308.

10. Cao B, Wang Y, Wen D, Liu W, Wang J, Fan G, et al. A trial of lopinavir-ritonavir in adults hospitalized with severe Covid-19. N Engl J Med. 2020;382(19):1787-99.

11. Jiang $H$, Deng $H$, Wang $Y$, Liu Z, Sun WM. The possibility of using Lopinave/ Litonawe (LPV/r) as treatment for novel coronavirus 2019-n Covpneumonia: a quick systematic review based on earlier coronavirus clinical studies. Zhonghua Jizhen Yixue Zazhi. 2020;29(2):182-6.

12. Khan PA, Nousheen BB, Maryam N, Sultana K. Middle east respiratory syndrome (MERS): a systematic review. Int J Pharm Sci Res. 2018;9(7):2616-25.

13. Ferron F, Subissi L, Silveira De Morais AT, Le NTT, Sevajol M, et al. Structural and molecular basis of mismatch correction and ribavirin excision from coronavirus RNA. Proc Nat Acad Sci. 2018;115(2):E162-E171.

14. Gautret $P$, Lagier JC, Parola P, Hoang VT, Meddeb L, Mailhe M, et al. Hydroxychloroquine and azithromycin as a treatment of COVID-19: results of an open-label non-randomized clinical trial. Int J Antimicrob Agents. 2020 Mar 20:105949. doi: 10.1016/j.ijantimicag.2020.105949. Online ahead of print.

15. Zhao ZW, Zhang FC, Xu M, Huang K, Zhong WN, Cai WP, et al. Clinical analysis of 190 cases of outbreak with atypical pneumonia in Guangzhou in spring, 2003. Zhonghua Yixue Zazhi. 2003;83(9):713-8.

16. Meng QH, Dong PL, Guo YB, Zhang K, Liang LC, Hou W, et al. Use of glucocorticoid in treatment of severe acute respiratory syndrome cases. Zhonghua Yufang Yixue Zazhi. 2003;37(4):233-5.

17. Schoggins JW, Wilson SJ, Panis M, Murphy MY, Jones CT, Bieniasz P, et al. A diverse range of gene products are effectors of the type I interferon antiviral response. Nature. 2011;472(7344):481-5.

18. Wan Y, Shang J, Graham R, Baric RS, Li F. Receptor recognition by novel coronavirus from Wuhan: An analysis based on decade-long structural studies of SARS. J Virol. 2020 Mar 17;94(7):e00127-20.

19. Fang L, Karakiulakis G, Roth M. Are patients with hypertension and diabetes mellitus at increased risk for COVID-19 infection? [published correction appears in Lancet Respir Med. 2020 May 18::]. Lancet Respir Med. 2020;8(4):e21. doi:10.1016/S2213-2600(20)30116-8

20. Al-Tawfiq JA, Al-Homoud AH, Memish ZA. Remdesivir as a possible therapeutic option for the COVID-19. Travel Med Infect Dis. 2020;34:101615.

21. Agostini ML, Andres EL, Sims AC, Graham RL, Sheahan TP, Lu X, et al. Coronavirus susceptibility to the antiviral remdesivir (GS-5734) is mediated by the viral polymerase and the proofreading exoribonuclease. mBio. 2018;9(2):e00221-18.
22. Gupta R, Ghosh A, Singh AK, Misra A. Clinical considerations for patients with diabetes in times of COVID-19. Diabetes Metab Syndr. 2020;14(3):211-2.

23. Huang C, Wang Y, Li X, Ren L, Zhao J, et al. Clinical features of patients infected with 2019 novel coronavirus in Wuhan, China. Lancet. 2020;395(10223):497-506. doi:10.1016/S0140-6736(20)30183-5

24. Chen N, Zhou M, Dong X, Ou J, Gong F, et al. Epidemiological and clinical characteristics of 99 cases of 2019 novel coronavirus pneumonia inWuhan, China: a descriptive study. Lancet. 2020;395(10223):507-513. doi:10.1016/S0140-6736(20)30211-7

25. Wang D, Hu B, Hu C, Zhu F, Liu X, et al. Clinical characteristics of 138 hospitalized patients with 2019 novel coronavirus-infected pneumonia inWuhan, China. JAMA. Published online February 7, 2020. doi:10.1001/ jama.2020.1585

26. Yang $X, Y u Y, X u J$, Shu $H, X i a ~ J$, et al. Clinical course and outcomes of critically ill patients with SARS-CoV-2 pneumonia inWuhan, China: a single-centered, retrospective, observational study. Lancet Respir Med. Published online February 24, 2020. doi:10.1016/S2213-2600(20)30079-5

27. Young BE, Ong SWX, Kalimuddin S, Epstein L, Killerby ME, et al; Singapore 2019 Novel Coronavirus Outbreak Research Team. Epidemiologic features and clinical course of patients infected with SARS-CoV-2 in Singapore. JAMA. Published online March 3, 2020. doi:10.1001/ jama.2020.3204

28. Kujawski SA,Wong K, Collins JP, et al. First 12 patients with coronavirus disease 2019 (COVID-19) in the United States. medRxiv. Preprint posted March 9, 2020. doi:10.1101/2020.03.09.20032896

29. Guan WJ, Ni ZY, Hu Y, Liang WH, Ou CQ, et al; China Medical Treatment Expert Group for Covid-19. Clinical Characteristics of Coronavirus Disease 2019 in China. N Engl J Med. Published online February 28, 2020. doi:10.1056/NEJMoa2002032

30. Tran K, Cimon K, Severn M, Pessoa-Silva CL, Conly J. Aerosol generating procedures and risk of transmission of acute respiratory infections to healthcare workers: a systematic review. PLoS One. 2012;7(4):e35797.

31. Hersberger L, Bargetzi L, Bargetzi A, Tribolet P, Fehr R, Baechli V, et al. Nutritional risk screening (NRS 2002) is a strong and modifiable predictor risk score for short-term and long-term clinical outcomes: secondary analysis of a prospective randomised trial. Clin Nutr. 2019:S02615614(19)33171-1.

32. Avendaño-Reyes JM, Jaramillo-Ramírez H. Prophylaxis for stress ulcer bleeding in the intensive care unit. Rev Gastroenterol Mex. 2014;79(1): 50-5.

33. Golding, John \& Wesnes, Keith \& Leaker, Brian. (2018). The effects of the selective muscarinic $M 3$ receptor antagonist darifenacin, and of hyoscine (scopolamine), on motion sickness, skin conductance \& cognitive function. Br J Clin Pharmacol. 2018;84(7):1535-43.

34. ARDS Definition Task Force, Ranieri VM, Rubenfeld GD, Thompson BT, Ferguson ND, Caldwell E, et al. Acute respiratory distress syndrome: the Berlin Definition. JAMA. 2012;307(23):2526-33.

35. Greffrath WP, Du Plessis JM, Viljoen M, Cockeran M. Hypertriglyceridaemia and the risk of pancreatitis six months post lopinavir/ritonavir initiation. S Afr J HIV Med. 2018;19(1):a766

36. Hwang JL, Weiss RE. Steroid-induced diabetes: a clinical and molecular approach to understanding and treatment. Diabetes Metab Res Rev. 2014;30(2):96-102.

37. Hirasawa $\mathrm{H}$, Oda $\mathrm{S}$, Nakamura M. Blood glucose control in patients with severe sepsis and septic shock. World J Gastroenterol. 2009;15(33):4132-6.

38. Singh AK, Singh A, Shaikh A, Singh R, Misra A. Chloroquine and hydroxychloroquine in the treatment of COVID-19 with or without diabetes: a systematic search and a narrative review with a special reference to India and other developing countries. Diabetes Metab Syndr. 2020;14(3):241-6

39. Baretic M. Case report of chloroquine therapy and hypoglycaemia in type 1 diabetes: What should we have in mind during the COVID-19 pandemic? Diabetes Metab Syndr. 2020;14(4):355-6.

40. Juurlink DN. Safety considerations with chloroquine, hydroxychloroquine and azithromycin in the management of SARS-CoV-2 infection. CMAJ. 2020;192(17):E450-E453. 